\title{
POOR MENTAL HEALTH INFLUENCES RISK AND DURATION OF UNEMPLOYMENT: A PROSPECTIVE STUDY
}

Peter Butterworth ${ }^{1}$, Liana S. Leach ${ }^{1}$, Jane Pirkis ${ }^{2}$, Margaret Kelaher ${ }^{2}$

${ }^{1}$ Centre for Mental Health Research, The Australian National University, Canberra, 0200, Australia

${ }^{2}$ Centre for Health Policy, Programs and Economics, The University of Melbourne, Melbourne, VIC, 3010, Australia

Corresponding author: Peter Butterworth. Phone: +61 02 61258097. Fax: +61 02

61250733. Email: Peter.Butterworth@anu.edu.au 


\begin{abstract}
Purpose: The current paper aims to investigate the role of mental health in determining future employment status. Much of the previous longitudinal and prospective research has focused on how unemployment adversely influences mental health, while the reverse causal direction has received much less attention.
\end{abstract}

Methods: This study uses five waves of data from 5,846 respondents in the HILDA survey, a nationally representative household panel survey conducted annually since 2001. Prospective analyses followed a group of respondents who were not unemployed at baseline across four subsequent years and investigated whether baseline mental health was associated with subsequent unemployment.

Results: Baseline mental health status was a significant predictor of overall time spent unemployed for both men and women. Decomposing this overall effect identified sex differences. For women but not men, baseline mental health was associated with risk of experiencing any subsequent unemployment whereas for men but not women mental health was associated with the duration of unemployment amongst those who experienced unemployment.

Conclusions: By following a group of respondents who were not unemployed over time we showed that poor mental health predicted subsequent unemployment. On average, men and women who experienced symptoms of common mental disorders spent greater time over the next four years unemployed than those with better mental health but there were sex differences in the nature of this effect. These findings highlight the importance of mental health in the design and delivery of employment and welfare policy. 


\section{Introduction}

There is ample research demonstrating that unemployment and poor mental health are related $[1,2]$. A large body of cross-sectional work has confirmed that unemployed individuals have poorer mental health than those who are employed. Dooley, Fielding and Levi reviewed this association and found that indicators of illness such as depression levels, psychiatric admissions and suicide were all correlated with unemployment [1]. The strongest evidence for a cross-sectional relationship has been shown using national population data. Wilhelm et al [3] found that being unemployed was the strongest correlate of Major Depressive Disorder (MDD) using data from the Australian National Survey of Mental Health and Well-being. Results from the National Comorbidity Survey in the US found unemployment was a prominent risk factor for both major and minor depression [4]. The UK National Survey of Psychiatric Morbidity similarly found unemployment was strongly associated with psychiatric disorder [5]. While the crosssectional association between unemployment and poor mental health is apparent, this information is limited in terms of the questions it can answer about causal interactions.

To address the limitations of cross-sectional research, a number of longitudinal studies have been conducted. However, this research has been largely one-directional, focusing on whether changes in employment lead to changes in mental health. Results have suggested there is evidence for this causal progression. Graetz [6] found that individuals who moved from unemployment into employment reported significant improvement in their general mental health. Such research is of particular interest to policy makers who wish to know whether employment programs and policies will result in socio-economic, 
social and health improvements. There is less research exploring the reverse causal progression; that is, the effect pre-existing mental health problems, particularly the common mental disorders such as anxiety and depression, may have on subsequent employment opportunities. Research conducted by Heponiemi et al. [7] is one of the few studies to have found evidence of selection into unemployment based on a history of mental illness, although only a crude measure of mental health was used (admission to hospital as a psychiatric inpatient). Using panel data from a US national survey, Dooley and colleagues [8] found that depression symptoms amongst adequately employed young adults (in their early 30s) predicted unemployment two years later. This is an important area for investigation given the well documented healthy worker effects in studies of physical health [9]. Selection based on health states is a central aspect of the temporal relationship between employment and health. At a population level it is vital to consider the full social and economic impact of mental disorders when quantifying disease. Further, at a personal level, if poor mental health is a barrier to employment, people may become entrenched in a cycle of disadvantage where the connection between poor health and exclusion from employment is reinforced over time [8].

An additional important and frequently neglected aspect of the relationship between employment circumstances and mental health is that both vary over time, such that periods of unemployment and/or poor health may come and go, and last for varying durations. Difficulties in obtaining appropriate longitudinal data have meant that temporal aspects of the relationship, such as recurrence and duration, have for the most part remained uninvestigated. Studies that have explored these factors have again 
examined the effects of duration of unemployment on mental health, rather than reversing the causal direction and exploring the role that health selection may play on duration of unemployment. These studies have shown that the long-term unemployed have poorer mental health than the short-term unemployed $[10,11]$.

The current study addresses an important gap in the research literature: the role mental health plays in selecting future employment status. Five waves of nationally representative longitudinal data from the Household Income and Labour Dynamics in Australia Survey are used to investigate the effect of baseline mental health on the subsequent experience of unemployment. Our initially analysis considers a measure of the number of time periods (a count measure which can readily be converted to a measure of weeks) unemployed over the following four years. However, the majority of respondents do not experience unemployment and have a value of 0 on this measure. This measure of overall average time spent unemployed can be decomposed into two different effects: i) the risk of any subsequent unemployment ( 0 vs. $1+$ periods unemployed), and ii) the duration of unemployment for those who do experience unemployment (a count measure that is always greater than 0 ). All of these constructs are potentially important and could reflect different aspects of the relationship between mental health and subsequent unemployment and we consider all three measures in the current paper.

\section{Methods}

This study reports a prospective analysis of data from five waves of the Household, Income and Labour Dynamics in Australia (HILDA) Survey (release 5.1). This is a 
nationally representative household panel survey conducted annually from 2001 . The survey was approved by the Human Research Ethics Committee at the University of Melbourne. The survey used a multi-stage sampling approach, sampling households within dwellings within a selection of administrative areas. For more detail see Wooden and Watson [12].

\section{Sample}

At baseline, there were 7,682 responding households (response rate of 66\%). Within households, 13,969 household members aged 15 years and over (92\% of the eligible population) completed a personal interview and $94 \%$ of these returned a self-completion questionnaire (SCQ). Given the focus on employment, this analysis is restricted to respondents aged between 20 and 50 at wave 1 so as to minimise the influence of agenormative transitions into and out-of the workforce. Further, as we are concerned with the direction of association leading from poor mental health to unemployment we also restricted the sample to those respondents who were not unemployed at the time of the wave 1 interview and had not been unemployed for a significant period (more than 20\%) of the preceding year (excluding 472 respondents). In addition, we excluded those respondents who at baseline reported that they could not work due to injury or ill health $(n=5)$ and those who did not participate in at least 3 waves of data collection $(15.4 \%)$. The final sample size, therefore, comprised 5,846 respondents.

\section{Measures}


Mental health at baseline/wave 1 was assessed using the Mental Health Inventory (MHI), a subscale from the SF-36 general health survey which was included in the SCQ . The MHI assesses symptoms of depression and anxiety (nervousness, depressed affect) and positive aspects of mental health (feeling calm, happy) in the past four weeks. Respondents indicate the frequency of symptoms over the past four weeks and responses are summed and transformed to a normalised scale from 0 to 100 . Previous research has demonstrated that the MHI has reasonable validity and is an effective screening tool able to identify common mental disorders in the community [13-15]. Analysis of baseline HILDA Survey data showed reasonable internal consistency (Cronbach's alpha $=0.82$ ). This analysis interprets the MHI as a measure of common mental health problems, reflecting the high comorbidity found between symptoms of anxiety and depression [16] and evidence that a higher-order factor represents commonality among internalising disorders [17]. For these analyses we use the reversed mental health scale score (a continuous scale where higher scores represent poorer mental health).

The outcome measure is the time each respondent spent unemployed during the four years following baseline measurement. During each interview, respondents were asked systematically about their labour force activity over the previous 12 months, including time spent working, unemployed and looking for work, and time out of the labour force. This information was used to construct a calendar representing labour force status at the beginning, middle and end of each month. There were, therefore, 36 measurement periods in each year. The outcome measure used in the current study represents a count of the number of these periods that each respondent reported being unemployed and looking 
for work across the survey period and can be readily converted to an estimate of days/weeks.

A range of covariates were considered, including several measures of income and social status and other factors demonstrated in previous research to be associated with mental health and employment status. Covariates included age, sex, partnered status (married/de facto vs no partner), presence of dependent children in the household, physical disability (derived from the physical functioning subscale of the SF-36), housing tenure, percent of post-education life in paid employment, occupational classification of current or previous job (classified into skill level), educational qualifications (tertiary degree, diploma/certificate, completed secondary school, not completed secondary school), experience of financial hardship, current smoking status, equivalised household income, and baseline labour force status (employed, not in labour force (NILF) due to ill health, NILF due to home duties, NILF other). Note that NILF excludes those who are unemployed and actively seeking work.

\section{Analytic Strategy}

Given gendered differences in labour-force activity, all analyses were conducted separately for men and women. Initial descriptive analyses considered the baseline sociodemographic characteristics of the sample and examined experience and duration of unemployment over the four subsequent waves. A preliminary analysis examined whether the association between baseline mental health and unemployment was consistent over the four years of follow-up. Negative binomial (NB) regression analyses 
were conducted (over-dispersion precluded use of poisson regression) on the count of periods of unemployment, with the continuous mental health measure the primary independent measure. An initial univariate model was complemented by a full model in which all covariates were included. As discussed, we also decomposed the initial NB analysis into two separate components, separately examining whether baseline mental health predicted i) the risk of any subsequent unemployment experience (a binary outcome evaluated with logit regression models) and ii) the duration of unemployment amongst those who reported being unemployed at some time (a count measure greater than 0 and assessed with a zero-truncated negative binomial regression (ZTNB) model). To aid interpretation of significant effects, regression parameters were used to contrast the predicted unemployment duration/likelihood of unemployment for respondents with good and poor mental health which we operationalised as scores at the 15th and 85th percentiles on the mental health scale (holding all other model parameters constant at their mean).

Data were weighted to reflect population characteristics, using the person-level weights (representing selection probabilities at wave 1) supplied with the HILDA Survey data. Cases with missing data (ranging from 0 to $0.56 \%$ of responses to individual items over waves) were excluded on an analysis-by-analysis basis. Analyses were conducted using Stata 10.0. 
Sensitivity analyses examined the robustness of the findings by considering only those respondents actively employed at wave 1, imputing unemployment duration for those with missing data, examining a wider age range and analyzing unweighted data.

\section{Results}

There were 2,686 men and 3,160 women included in the analysis. Table 1 presents baseline (weighted) characteristics of the sample, separately for men and women. Overall, $16.6 \%$ of men and $20.6 \%$ of women reported at least one time period on the HILDA Survey employment calendar as unemployed. On average, men reported 2.5 periods unemployed (approximately 3.6 weeks) and women reported 3.8 periods of unemployment (5.5 weeks). Restricting the analysis to those who did report some time of unemployment, the average duration of unemployment over the four years was 21.9 weeks for men and 26.6 weeks for women. In addition, 53\% of these men and $58 \%$ of these women reported over 13 weeks (or 3 months) of the following four years unemployed.

Initial NB models considered whether the association between baseline mental health differed across subsequence years (regressing unemployment on mental health, year (1 to 4), and the year by mental health interaction terms). The model showed a significant effect of mental health but none of the year or year-by-mental health interaction terms was significant. Further, removing the interaction terms from the model did not significant alter overall model fit $\left(\chi^{2}=0.39, d f=3, p=.94\right)$. 
Table 2 presents the results for the series of regression models testing the association between mental health and subsequent employment outlined above (reporting coefficients and standard errors). The univariate results are presented in the upper panel of the table for men and women separately. The initial NB regression models show that the relationship between baseline mental health scores and the count of periods of unemployment was significant in simple analysis for both men (coefficient $=0.21)$ and women $($ coefficient $=0.14)$. Higher mental health scores $($ worse mental health) were associated with increased periods of time unemployed over the following four years. This is important as none of the respondents were unemployed at baseline. The model parameters suggest that, on average, men with poor mental health (at the 85th percentile) spent 5.1 weeks of the next four years unemployed compared with 2.4 weeks for those with better mental health (at the 15th percentile). The corresponding figures for women were 6.7 and 4.1 weeks.

When we decompose the NB models into two components we observe that, for men, poorer mental health is associated with increased risk of any subsequent unemployment but is somewhat more strongly associated with increased duration of unemployment for those who were unemployed at some time. The parameters of the logit regression model predict that $19.1 \%$ of those with poor mental health experience subsequent unemployment compared with $14.6 \%$ of those with better mental health. The ZTNB model shows that those men with poor mental health who experience unemployment are unemployed for 24.8 weeks compared to 14.8 weeks for those with better mental health. The results for women indicate that poorer mental health at baseline is associated with an 
increased likelihood of experiencing subsequent unemployment but not with the duration of unemployment. The prediction from the logit regression model is that $24.3 \%$ of women with poor mental health become unemployed compared with $16.5 \%$ of women with better mental health.

The lower panel of Table 2 replicates these regression models for men and women while including covariates. Overall, the effect of baseline characteristics was reasonably consistent for men and women. The NB models showed that low occupational skill level, lesser periods of adult life in paid employment, not being active in the labour force at baseline, experience of financial hardship, and current smoking status were all associated with greater periods of subsequent unemployment. For men, the presence of dependent children and not being partnered were also correlates of subsequent unemployment, though these measures were not significant predictors of women's subsequent unemployment. For women, poorer physical health and rental housing tenure were also associated with duration of unemployment.

In terms of mental health, the results of the full NB models show that, when compared to the univariate models, some of the predictive power of mental health is explained by the inclusion of the covariates. However, baseline mental health remained a significant predictor of time unemployed for both men (0.11) and women (0.09). The predicted time spent unemployed by respondents at the 15 th and 85 th percentiles on the mental health scale was 1.9 and 2.9 weeks for men and 3.0 and 4.2 weeks for women. For men, decomposing the NB models showed that, having controlled for other relevant factors, 
mental health was not a significant predictor of the experience of unemployment (in the logit regression model). However, mental health was associated with the duration of unemployment (estimated difference of 15.4 vs 21.0 weeks). Consistent with the univariate results, mental health was associated with women's risk of unemployment (estimated $16.2 \%$ vs $19.6 \%$ ) but not with the duration of unemployment for those who do experience unemployment.

A number of sensitivity analyses examined the robustness of the current findings. Analysis restricted to those respondents who employed at baseline (i.e, excluding those not participating in the workforce: number of men reduced from 2,686 to 2,527 and women from 3,160 to 2,370 ) showed the same, if slightly stronger, associations between mental health and subsequent unemployment. Broadening the age range from 50 to 55 years and from 50 to 60 years replicated the results, though the effect of mental health for men in the NB model with covariates was marginally significant $(p=.055)$. Some respondents included in the analysis did not participate in all waves of data collection. This limits the number of periods of time that they could possibly spend unemployed. We repeated the initial analyses limited to those respondents who participated in all 5 waves, and also re-weighting the data from those who participated in less than 5 waves to estimate their period of unemployment across all possible waves. Both of these approaches produced identical results to those reported in the current analysis. Finally, analysis of unweighted data also showed that same pattern of results though again the effect of mental health for men in the NB model with covariates was marginally significant $(p=.053)$. 


\section{Discussion}

The current study supports the notion that, even when considering the distribution of anxiety and depression within the general community, those with poorer mental health are selected into unemployment. After controlling for other relevant factors, poorer baseline mental health was found to be associated with greater time spent unemployed for both men and women. Decomposing this overall effect showed that poorer baseline mental health was significantly associated with increased risk of experiencing any subsequent unemployment for women but for men was associated with greater duration of unemployment.

\section{Contribution to the literature}

The current study offers a number of important findings not previously available in the literature. Differences in the findings based on whether the outcome of interest was average overall time unemployed, any experience of unemployment, or unemployment duration for those who are unemployed, are of note. Overall, the experience of any unemployment was not uncommon, with about one in five men and women experiencing at least one period of unemployment over the four years. However, it is also evident that poor mental health, indicated by symptoms of anxiety and depression, increases an individuals' time in unemployment.

A number of covariates were found to be associated with subsequent number of periods of unemployment for both men and women, including low occupational skill level, lesser 
periods of adult life in paid employment, not being active in the labour force at baseline, experience of financial hardship, and current smoking status. These findings are consistent with previous research that has shown associations between poor socioeconomic status and unemployment [18], and smoking and unemployment [19]. However, many of these covariates were found to be differently associated with risk of any unemployment and duration of unemployment, evident from closer examination of the logit and ZTNB models. For example, physical health (for men) was significant in the ZTNB models but not in the logit models. This factors therefore, seem to be associated with duration of unemployment but not the likelihood of experiencing unemployment. This makes sense intuitively as physical health problems can be considered long-term problems in themselves, with long-term effects. In contrast, rental housing tenure (for men) and occupational skill level (for women) were only significant in the logit and not the ZTNB models, suggesting that these factors are linked with the risk of unemployment but not its duration. It was also evident that a null effect of age in the NB models for both men and women reflected the combination of opposite effects, with increasing age associated with a declining risk of experiencing unemployment but balanced by increased duration of unemployment for those who did become unemployed.

Previous prospective research linking mental illness to poor employment prospects has focused on respondents with severe and low prevalent psychiatric disorders or in-patient admissions [7]. This study makes an important contribution as it uses a continuous measure of mental health symptoms (MHI-5) from a representative community survey. Our analyses evaluated a linear effect between the MHI scale score and risk/duration of 
subsequent unemployment. Further, the study followed a cohort of adults who were not unemployed at baseline, had not been unemployed for any substantial period of the prior 12 months, and who reported that injury/ill health did not prevent them from working. We are, therefore, capturing an effect of mental health in the general population and, as such, argue that the findings have broader implications than previous work.

\section{Gender differences}

There were important gender differences in the pattern of associations between mental health and subsequent unemployment. For women, the significant effect of mental health on subsequent unemployment reflected an increased risk of experiencing any unemployment and not the subsequent duration of that unemployment. Nonetheless, much of the strong univarate relationship between mental health and risk of unemployment in women was explained by factors such as employment status at baseline and strength of prior workforce connection, as well as general measures of socioeconomic disadvantage such as experience of hardship and rental housing. This suggests that, in part, more general disadvantage in terms of labour force skills and experience and socio-economic adversity that explains much of women's subsequent risk of unemployment. These findings might also reflect the type of work women are likely to enter into (lower skilled, casual or part-time work), given they are not typically the primary earner in couples and families. However, mental health remains a significant independent factor leading to increased risk of unemployment and, overall, women with poorer mental health at baseline reported more periods of time unemployed than those with better mental health. 
In contrast, for men the significant overall effect of poorer mental health on subsequent unemployment was largely driven by greater duration of unemployment for those who experienced unemployment rather than an increased predisposition to experience unemployment. The modest univariate finding of an increased risk of experiencing unemployment among men with poor mental health was explained by occupational skill level, smoking status, prior employment status, rental housing and not having a partner. However, those men with poorer mental health who became unemployed remained unemployed for a longer period than those with better mental health and this effect was not explained by their socio-economic, employment or personal circumstances. Thus, for men, poor mental health seems a barrier that reduces their likelihood of returning to the workforce.

The current findings identify gender differences in the employment consequences of mental illness, and complement the substantial body of previous research which shows that unemployment and movements in and out of the labour force have different impacts on men and women because of the gendered nature of work and family responsibilities. For example, research conducted by Artazcoz et al and Dalgard et al [20, 21] found that the association between unemployment and poor mental health was stronger for men than for women. Other research [22] has found that negative experiences involving work are reported more often by men than women, a factor which is associated with greater depression and anxiety. The current findings suggest that mental health also has a 
gendered effect on subsequent employment outcomes and points to a need for more research to disentangle selection and causation in this association.

\section{Policy and Practical Implications}

The current study has implications for the development of workforce policy. A common component of many governments' social inclusion, participation and economic agendas is finding work for the unemployed. The Australian Government report Social Inclusion: Origins, Concepts and Key Themes names 'securing a job' as a key component of social inclusion [23]. The current findings show that, at a population level, experiencing symptoms of psychological distress increases people's likelihood of subsequent unemployment and/or duration of unemployment. Action has been taken with the implementation of workplace programs designed to assist people with mental health problems remain in the workforce (e.g. Work Outcomes Research and Cost Benefit (WORC) Project [24] and beyondblue National WorkPlace Program [25]) or to support people with mental illness into the workforce (e.g., reform of Disability Employment Services [26]) . Overall, the finding that poor mental health increases the average time spent unemployed for both men and women suggests a focus on addressing mental health

problems in mainstream employment programs. However, evidence that mental health is associated with increased risk of unemployment for women but increased duration out of the workforce for men who experience unemployment may suggest gendered differences in the aims and approaches of these duration of not only the risk of unemployment but also duration of unemployment (particularly for men), 
The current findings also provide support for general community-based programs that have been introduced to address high prevalence disorders - e.g. Better Outcomes in Mental Health Care in Australia [27] and Improving Access to Psychological Therapies in the UK [28]. While these programs have been principally designed to reduce symptom levels in the general public, and do not have reducing unemployment as part of their remit, out findings suggest a subsidiary benefit is likely to be increased employment, and therefore an overall reduction in the societal costs of mental health problems.

\section{Limitations}

Several potential limitations should be considered. First, the current study was not designed to evaluate the causation theory of unemployment and mental health, that is, the idea that changes in employment lead to changes in mental health. While our findings show that people with mental health problems are selected into greater time unemployed, this does not mean the reverse, that greater periods of unemployment leads to mental health problems, is not untrue. Indeed, previous discussions and investigations aimed at untangling the causal direction of the association, suggest effects occur in both directions $[29,30]$. Second, we were limited to the scales available in the HILDA Survey and, therefore, could not measure other diagnostic aspects of mental health illness. This means that while associations between measures of poor general mental health and unemployment were identified, specifics about the relationship between diagnostic mental illnesses (e.g. Major Depressive Disorder, Bipolar Disorder) and unemployment cannot be commented on. Our analysis was restricted to respondents of prime working age, which we defined as 20 to 50 years at baseline. This was to avoid potential influence on the current results of the health correlates of normative transitions into and 
out of the workforce (e.g., [31]). While sensitivity analyses confirmed that the results held for a wider age range, future research should focus on the association between mental health and subsequent unemployment among younger adults, to coincide with the peak in the onset of depression and transition into the workforce [10] and amongst older adults to better inform policy focused on encouraging older workers to delay retirement [see 32]. Finally, the current analysis was restricted to the first five waves of data from the HILDA Survey to reflect a relatively short time frame similar to many previous studies (e.g., [8]). While we found no evidence that the effect of baseline mental health on unemployment duration was moderated by time, it is important for future research to consider the longer-term consequences of mental health using data from a longer study period when available.

\section{Conclusions}

The current study shows that poor mental health is associated with subsequent unemployment in a representative community sample of Australian adults. Women who experience symptoms of common mental disorders on average spend greater time unemployed than those with better mental health, while for men poor mental health predicts greater duration of unemployment. These findings highlight the importance of general mental health in the design and delivery of employment and welfare policy. 


\section{Acknowledgments}

The HILDA survey was initiated and is funded by the Commonwealth Department of Families, Housing, Community Services and Indigenous Affairs (FaHCSIA) and is managed by the Melbourne Institute of Applied Economic and Social Research (MIAESR). The findings and views reported in this report, however, are those of the authors and should not be attributed to either FaHCSIA or the MIAESR. This work was supported by the National Health and Medical Research Council Career Development Award 525410 to $\mathrm{PB} ;$.....???????. 


\section{References}

1 Dooley D, Fielding J, Levi L (1996) Health and unemployment. Annu Rev Public Health 17:449-65.

2 Fryers T, Melzer D, Jenkins R (2003) Social inequalities and the common mental disorders. Social Psychiatry and Psychiatric Epidemiology 38:229-37.

3 Wilhelm K, Mitchell P, Slade T, et al (2003) Prevalence and correlates of DSM-IV major depression in an Australian national survey. J Affect Disord 75:155-62.

4 Kessler RC, Zhao S, Blazer DG, et al (1997) Prevalence, correlates, and course of minor depression and major depression in the National Comorbidity Survey. J Affect Disord 45:19-30.

5 Jenkins R, Lewis G, Bebbington P, et al (2003) The National Psychiatric Morbidity Surveys of Great Britain--initial findings from the Household Survey. Int Rev Psychiatry 15:29-42.

6 Graetz B (1993) Health consequences of employment and unemployment: longitudinal evidence for young men and women. Soc Sci Med 36:715-24.

7 Heponiemi T, Elovainio M, Manderbacka K, et al (2007) Relationship between unemployment and health among health care professionals: health selection or health effect? J Psychosom Res 63:425-31.

8 Dooley D, Prause J, Ham-Rowbottom KA (2000) Underemployment and depression: Longitudinal relationships. J Health Soc Behav 41:421-36.

9 Arrighi HM, Hertz-Picciotto I (1994) The Evolving Concept of the Healthy Worker Survivor Effect. Epidemiology 5:189-96.

10 Mossakowski KN (2009) The Influence of Past Unemployment Duration on Symptoms of Depression Among Young Women and Men in the United States. Am J Public Health 99:1826-32.

11 Stankunas M, Kalediene R, Starkuviene S, et al (2006) Duration of unemployment and depression: a cross-sectional survey in Lithuania. BMC Public Health 6:174.

12 Wooden M, Watson N, eds (2002) The Household, Income and Labour Dynamics in Australia (HILDA) Survey: an introduction. University of Melbourne, Melbourne.

13 Butterworth P, Crosier T (2004) The validity of the SF-36 in an Australian National Household Survey: demonstrating the applicability of the Household Income and Labour Dynamics in Australia (HILDA) Survey to examination of health inequalities. BMC Public Health 4:44.

14 Rumpf HJ, Meyer C, Hapke U, et al (2001) Screening for mental health: validity of the MHI-5 using DSM-IV Axis I psychiatric disorders as gold standard. Psychiatry Res 105:243-53.

15 Ware JE, Jr., Gandek B (1998) Overview of the SF-36 Health Survey and the International Quality of Life Assessment (IQOLA) Project. J Clin Epidemiology 51:903-12. 
16 Mineka S, Watson D, Clark LA (1998) Comorbidity of anxiety and unipolar mood disorders. Annu Rev Psychol 49:377-412.

17 Krueger RF, Caspi A, Moffitt TE, et al (1998) The structure and stability of common mental disorders (DSM-III-R): a longitudinal-epidemiological study. J Abnorm Psychol 107:216-27.

18 Bartley M, Owen C (1996) Relation between socioeconomic status, employment, and health during economic change, 1973-93. Br Med J 313:445-9.

19 Hammarstrom A, Janlert U (2003) Unemployment -- an important predictor for future smoking: a 14-year follow-up study of school leavers. Scand J Public Health 31:22932.

20 Artazcoz L, Benach J, Borrell C, et al (2004) Unemployment and mental health: understanding the interactions among gender, family roles, and social class. Am $\mathbf{J}$ Public Health 94:82-8.

21 Dalgard O, Dowrick C, Lehtinen V, et al (2006) Negative life events, social support and gender difference in depression. Soc Psychiatry Psychiatr Epidemiol 41:444-51.

22 Leach LS, Christensen H, Mackinnon AJ, et al (2008) Gender differences in depression and anxiety across the adult lifespan: the role of psychosocial mediators. Soc Psychiatry Psychiatr Epidemiol 43:983-98.

23 Hayes A, Gray M, Edwards B (2008) Social Inclusion: Origins Concepts and Key Themes. Australian Institute of Family Studies, prepared for the Social Inclusion Unit, Department of the Prime Minister and Cabinet, Canberra.

24 beyondblue. Work Outcomes Research and Cost Benefit (WORC) Project. http://www.beyondblue.org.au/index.aspx?link_id=4.75

25 beyondblue. National Workplace Progam. http://www.beyondblue.org.au/index.aspx?link_id=4.1028

26 Department of Education, Employment and Workplace Relations (2008). The Future of Disability Employment Services in Australia: Discussion paper. Commonwealth of Australia: Canberra.

27 Australian Department of Health andAgeing. Better Outcomes in Mental Health Care Program.

http://www.health.gov.au/internet/mentalhealth/publishing.nsf/Content/betteroutcomes-mental-health-care-1

28 UK Health Department. Improving Acess to Psychological Therapies Program.http://www.dh.gov.uk/en/Healthcare/Mentalhealth/Psychologicaltherapies/in dex.htm

29 Bartley M (1994) Unemployment and ill health: understanding the relationship. J Epidemiol Community Health 48:333 - 7.

30 Leach LS, Butterworth P, Strazdins L, et al (2010) The limitations of employment as a tool for social inclusion. BMC Public Health 10:621. 
31 Butterworth P, Gill S, Rodgers B, Anstey KJ, Villamil E, Melzer D. Retirement and the mental health of older Australians: analysis of the National Survey of Mental Health and Wellbeing. Soc Sci Med. 2006;62:1179-91.

32 Olesen SC, Butterworth P, Rodgers B. (in press) Is poor mental health a risk factor for retirement? Findings from a longitudinal population survey. Soc Psychiatry Psychiatr Epidemiol. 
Table 1: Baseline characteristics

\begin{tabular}{|c|c|c|}
\hline & Men & Women \\
\hline (mean, se) & $\begin{array}{l}35.9 \\
(.20)\end{array}$ & $\begin{array}{l}35.8 \\
(.19)\end{array}$ \\
\hline Dependent children in household & $40.3 \%$ & $49.2 \%$ \\
\hline Partnered (married or de facto) & $65.8 \%$ & $69.7 \%$ \\
\hline Physical health problems (SF-36) & $7.6 \%$ & $9.7 \%$ \\
\hline Occupational category & $14.0 \%$ & $19.7 \%$ \\
\hline $\begin{array}{r}\text { Tertiary qualifications } \\
\text { Not complete high school }\end{array}$ & $\begin{array}{l}23.1 \% \\
21.4 \%\end{array}$ & $\begin{array}{l}25.1 \% \\
30.7 \%\end{array}$ \\
\hline Housing tenure (renting) & $27.2 \%$ & $28.7 \%$ \\
\hline Previous work history (mean $\%$ of adult life, se) & $\begin{array}{l}89.1 \\
(.50)\end{array}$ & $\begin{array}{l}72.8 \\
(.60)\end{array}$ \\
\hline $\begin{array}{r}\text { Employed } \\
\text { Not in labour-force - ill }\end{array}$ & $\begin{array}{l}94.1 \% \\
1.1 \%\end{array}$ & $\begin{array}{l}75.7 \% \\
0.8 \%\end{array}$ \\
\hline Financial hardship & $13.3 \%$ & $12.8 \%$ \\
\hline $\begin{array}{l}\text { Equivalised household income } \\
\text { Smoker }\end{array}$ & $\begin{array}{l}\$ 41,000 \\
29.3 \%\end{array}$ & $\begin{array}{l}\$ 38,000 \\
25.1 \%\end{array}$ \\
\hline $\begin{array}{l}\text { Unemployment measures } \\
\text { Overall number of time periods unemployed } \\
\text { (mean, se, range) } \\
\text { Any experience of unemployment }\end{array}$ & $\begin{array}{l}2.51 \\
(0.18) \\
0-108 \\
16.6 \%\end{array}$ & $\begin{array}{l}3.78 \\
(0.24) \\
0-137 \\
20.6 \%\end{array}$ \\
\hline $\begin{array}{l}\text { Time periods unemployed for those who were } \\
\text { unemployed (mean, se, range) }\end{array}$ & $\begin{array}{l}15.11 \\
(0.81) \\
1-108\end{array}$ & $\begin{array}{l}18.36 \\
(0.92) \\
1-137\end{array}$ \\
\hline
\end{tabular}


Table 2: Prospective regression models predicting count of periods of unemployment (negative binomial), experience of any unemployment (logit regression) and duration of employment (zero-truncated negative binomial regression), presenting coefficients and standard errors separately for men and women.

\begin{tabular}{|c|c|c|c|c|c|c|}
\hline \multirow[b]{2}{*}{ SIMPLE MODELS } & \multicolumn{3}{|c|}{ Men } & \multicolumn{3}{|c|}{ Women } \\
\hline & $\begin{array}{l}\text { Overall count } \\
\text { Negative Binomial }\end{array}$ & $\begin{array}{c}\text { Any vs None } \\
\text { Logit }\end{array}$ & $\begin{array}{c}\text { Duration } \\
\text { Zero-trunc neg bin }\end{array}$ & $\begin{array}{l}\text { Overall count } \\
\text { Negative Binomial }\end{array}$ & $\begin{array}{c}\text { Any vs None } \\
\text { Logit }\end{array}$ & $\begin{array}{c}\text { Duration } \\
\text { Zero-trunc neg bin }\end{array}$ \\
\hline Mental Health (scale score) & $0.21(.04) * * *$ & $0.09(.04) *$ & $0.14(.03) * * *$ & $0.14(.03) * * *$ & $0.14(.03) * * *$ & $0.04(.03)$ \\
\hline
\end{tabular}

\section{FULL MODELS}

Mental Health (scale score)

$0.11(.05) *$

$0.02(.05)$

$0.09(.03) * *$

$0.09(.04) *$

$0.06(.03) *$

$0.02(.03)$

Education (tertiary degree)

$$
\text { Diploma/certificate }
$$

Completed high school

Not completed high school

Dependent children

$-0.16(.24)$

$-0.22(.28)$

$-0.00(.20)$

$0.06(.28)$

$-0.14(.26)$

$0.51(.20) *$

$-0.72(.21) * * *$

$-0.06(.23)$

Partnered (marriage/de facto)

Rental housing tenure

$0.30(.17)$

$0.31(.17)$

$-0.56(.17) * * *$

$-0.17(.15)$

$-0.21(.19)$

$0.05(.19)$

$0.13(.15)$

$-0.25(.15)$

$0.41(.14) * *$

$-0.01(.12)$

$-0.01(.11)$

$-0.02(.01)$

$0.02(.01) *$

Physical health (score/100)

Low skill occupation

$-0.76(.45)$

$0.49(.36)$

$0.76(.22) * * *$

$0.39(.17) *$

$-0.84(.26) * * *$

$-1.06(.46) *$

$-0.65(.36)$

Labour force status (employ) NILF (other)

NILF home duties NILF ill health

Financial hardship (scale)

Household income (equiv)

$\begin{array}{ll}1.15(.27) * * * & 0.95(.27) * * * \\ 1.46(.38) * * * & 1.56(.54) * * \\ 1.36(.44) * * & 1.31(.51) * * \\ 0.24(.11) * & 0.13(.11) \\ -0.01(.02) & -0.02(.03) \\ 0.53(.16) * * * & 0.28(.14) *\end{array}$

$1.15(.27) * * *$

$1.46(.38) * * *$

$1.36(.44) * *$

$-0.01(.02)$

$0.53(.16) * * *$
$0.52(.13) * * *$

$-0.23(.28) *$

$$
\begin{aligned}
& 0.42(.20) * \\
& 0.76(.29) * * \\
& 0.45(.19) * \\
& 0.08(.07) \\
& -0.02(.02) \\
& 0.23(.11) *
\end{aligned}
$$

$0.12(.16)$
$0.29(.15)$
$0.03(.16)$
$0.14(.12)$
$-0.23(.12)$
$0.35(.12) * *$
$-0.04(.01) * * *$
$0.07(.31)$
$0.25(.13) *$
$-0.34(.21)$

$0.20(.14)$

$0.09(.14)$

$0.12(.14)$

$0.09(.10)$

$-0.03(.11)$

$0.25(.10) *$

$0.02(.01) * *$

$-0.47(.26)$

$0.13(.10)$

$-0.51(.17) * *$

$1.23(.22) * * *$

$0.83(.14) * * *$

$0.91(.55)$

$0.23(.09) * *$

$0.00(.02)$

$0.14(.12)$
$0.40(.15) * *$

$0.50(.11) * * *$

$-0.10(.26)$

$0.11(.05) *$

$-0.00(.01)$

$0.18(.09)$

$* \mathrm{p}<.05, * * \mathrm{p}<.01, * * * \mathrm{p}<.001$ 